\title{
Reflections on the Israeli palestinian situation through the eyes of self psychology theory
}

\section{Opinion}

The Israeli-Palestinian conflict has persisted for several decades. Part of this reality is that from one military clash to another, acts of violence are experienced by both sides. This short essay seeks to understand this unfortunate situation through the lens of self psychology theory. Self psychology is a psychoanalytic theory that was founded in the seventies of the previous century by Heinz Kohut, an American psychoanalyst. It was Kohut who developed the theoretical and clinical importance of empathy. Empathy is the ability to understand others from one's own perspective, to "put yourself into the shoes of, think yourself appropriately into the inner life of another person". ${ }^{1}$ Empathy, which is to be distinguished from love, kindness, or pity, could be, according to self psychology, the major force toward change and cure. Through empathic immersion with his patients, Kohut discovered the significant need for an individual's self to be understood deeply by others who are attuned to him (these others are called self-objects). Theseself-objects will acknowledge and affirm one's own psychological existence and uniqueness.

When a child develops without significant others who are strongly immersed with him emphatically, and do not recognize and affirm his uniqueness and abilities (mirror needs), a range of responses tend to arise in the child and later on, in the adult. One of them is narcissistic rage. ${ }^{2}$ Such rage emerges because the other, whom we depend upon and feel must recognize us and understand our needs (e.g., the self-object) refuses to assume that role. This leaves us with frustrated, unseen needs.In the Israeli-Palestinian conflict, each side has an unmistakable need - to be acknowledged and mirrored by the other side. When such acknowledgment is not provided, the result is an outright display of continuous frustration. Kohut. ${ }^{2}$ discusses the ramifications of a situation in which the self feels ostracized and oppressed. The need to be recognized gets magnified when denied and thus can burst forth violently - not only in the lives of individuals, but also in groups- pursuing omnipotence experience and grandiose aspirations. These are the cases when catchwords common to some declarations made by radical groups like Hamas or ISIS, who speak of converting everyone in the world to religious Muslims, or the ability to completely destroy the Zionist enemy occurs.

According to self psychology, the need to take revenge, rectify injustice, and erase insult at all costs, and the unrelenting compulsion that is deeply rooted toward these goals that does not give any respite to the recipient of the narcissistic blow, are the typical components of narcissistic rage. ${ }^{2}$ Kohut, as a psychoanalyst and therapist, came to understand that unsatisfied human needs lie behind this type of rage. That is, he understood aggression, in many instances, as a sign of an injured self, an insult. This insult must be addressed in a therapeutic setting in order to begin the curative process of relieving the pain and hurt that lies behind the rage. Only then is it possible that the grandiose demands will attenuate; the ability to acknowledge others and their subjectivity increase, and the ability to see and accept the negative and positive sides of others begin to develop. The therapeutic situation is quite different from the situation that exists between

\author{
Volume 6 Issue I - 2018
}

\author{
Shai Levinger \\ Graduate School of Creative Arts, University of Haifa, Israel
}

Correspondence: Shai Levinger, Graduate School of Creative Arts, University of Haifa, I99 Aba Khoushy Ave., Haifa 3498838, Israel,Tel +972--52-54 I-6463, Email shailevin@biu.013.net.il

Received: September 06, 2017 | Published: January 10, 2018

nations, especially nations in a state of continual conflict and hostility. Yet, self psychology challenges us to see nations as having selves with ambitions and ideals, the two most important components of the self., ${ }^{3,4}$ Groups and group-selves, as Kohut termed them, need other nations from outside as well as various types of leadership figures within the group to acknowledge the uniqueness, aspirations and ideals of the group.

From this perspective, the question arises as to whether each entity, and especially each type of leadership, can truly acknowledge the aspirations, uniqueness and even vulnerability of the other that lies behind the rage. So far it seems that this is only partially feasible. Over the last decade, inconsistent attempts have been made to give expression to the acknowledgements of the other side. For example, in the Bar Ilan speech, ${ }^{5}$ Israel's prime minister spoke of the two state solution, stating that " $\ldots$ the fundamental condition for ending the conflict is the public, binding and sincere Palestinian recognition of Israel as the national homeland of the Jewish People". This could be understood as the psychological need (not only the security-existence need) for acknowledgment of the uniqueness of the Israeli state, or group-self, as self psychology would term. Later in this speech, Netanyahu mentions "the need for us to recognize their rights." On the Palestinian side, Abu Mazen, the current President of the Palestinian Authority, addresses the Holocaust, saying it was "the most heinous crime to have occurred against humanity in the modern era". ${ }^{6}$ These are examples of attempts to show empathy and acknowledge the psychological existence, unique being and unique suffering that the other side experienced. Although there were attempts made at recognition and acknowledgement of needs, thus far both sides have often dismissed or related scornfully to such statements and acknowledgements made in the past. Thus, the feeling of hurt and lack of acknowledgment remains.

From the self psychology perspective, radical and violent groups emerge from people who have experienced hurt and unfulfilled needs in their lives, and this melds into a hurt and un-mirrored group-self, or feelings of hurt on a national level. The result is an outburst of narcissistic rage that is unsatisfied, and whose magnitude 
is comparable to the magnitude of a deeply injured self. This rage, in its chronic state, can fill the individual or group with grandiose goals and unrelenting omnipotence creating feelings of hurt that continue to eat away at any perceived positive traits possessed by the out-group.

Finally, while elaboration on this topic is beyond the scope of this brief opinion paper, it is important to consider leaders as self-objects to their own group. To what extent there are there leaders (e.g., political, art, or social leaders) within each group who affirm and support the uniqueness, ideals, and suffering of their own group?

Such mirroring, according to self psychology, supports group-self' growth, and thus mitigate narcissistic rage. For example, how is pride in Palestinians culture mirrored or celebrated by their leaders? Are the leaders of Do Palestinians and Israelis leaders develop and cherish unique cultural aspirations? To what extent are aspects of the group's history, its unique cultural heritage and strivings are reflected in order tothe development of the group-self? Following a self psychological frame, leaders can might be able to enhance the psychological groupself-identity growth in such ways that, subsequently reducing reduce narcissistic rage and thus of violent acts.

In this short opinion piece I have pondered about some ways in which self psychology can illuminate the Israeli-Palestinian conflict. A central point is the importance of leaderships to serve as empathic self-objects that can "see" the injured group-self, the unique aspirations and ideals of their own group-self as well as aspirations and ideals of other group-selves. Serving as such empathetic selfobjects might promote the development and growth of each group- self while mitigating anger and narcissistic rage which so often leads to acts of violence.

\section{Funding}

None.

\section{Acknowledgments}

None.

\section{Conflicts of interest}

The author declares there are no conflicts of interest.

\section{References}

1. Kohut H. On Empathy. International Journal of Psychoanalytic Self Psychology. 2010;5(2):122-131

2. Kohut $\mathrm{H}$. Thoughts on narcissism and narcissistic rage. Psychoanalytic Study of the Child. 1972;27(1):360-400.

3. Kohut H. Self Psychology and the Humanities. W.W. Norton and Company, USA. 1985.

4. Wolf E. Treating the self. Elements of clinical self psychology. The Guilford Press: USA;1988. 1-194.

5. Haaretz. Full text of Netanyahu's foreign policy speech at Bar Ilan. 2009

6. Rudoren J. Mahmoud Abbas Shifts on Holocaust. USA. 2014. 